\title{
Nanophotonic Emission Control for Improved Photovoltaic Efficiency
}

\author{
Julia S. van der Burgt and Erik C. Garnett*
}

Cite This: https://dx.doi.org/10.1021/acsphotonics.0c00152

Read Online

ABSTRACT: With the necessary transition to renewable energy at hand, there is a renewed research focus on increasing solar cell efficiency in order to reduce the cost of electricity. Nanomaterials are promising candidates to contribute to a new generation of low cost and highly efficient solar cells. Due to their wavelength-scale dimensions, nanomaterials display exceptionally strong lightmatter interactions that lead to large perturbations in absorption and emission compared to their bulk counterparts. Although most work on nanostructured solar cells has focused on increasing the
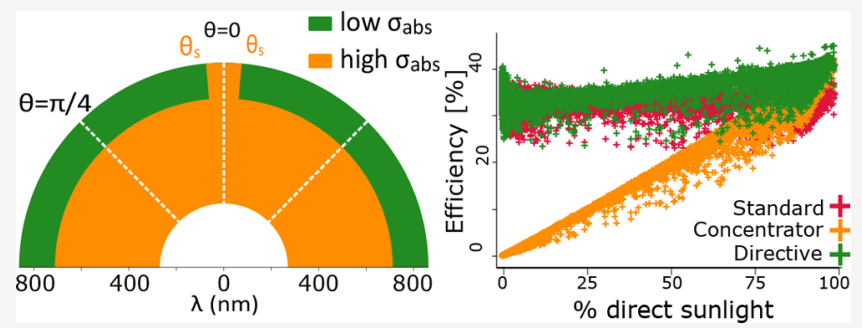
absorption, emission control may have even greater potential for improving efficiency of state-of-the-art solar cells. In this Perspective, we describe how nanostructures can be applied to improve solar cell efficiency, focusing on emission control. First, we analyze the requirements for making the most efficient solar cell by looking at the thermodynamics of energy conversion. We show that an ideal solar cell at open circuit displays emission that is identical to its absorption. Comparing this to the emission of a typical silicon solar cell shows that there are three differences: the intensity, the angles in which light is emitted, and the spectrum. These differences lead to a reduction in efficiency, mainly due to a drop in open circuit voltage. For each loss mechanism, we discuss how nanomaterials can manipulate the emission and thereby reduce the voltage loss. Finally, we analyze the performance of two conceptual designs for solar cells based on nanomaterials. These give a large improvement in efficiency compared to conventional designs, showing the great potential of nanomaterials in solar cells.

KEYWORDS: solar cells, nanomaterials, Shockley-Queisser limit, thermodynamic limit, emission control, directivity

$\mathrm{O}$ ver the past decades, the price of electricity generated by solar panels dropped drastically. ${ }^{1}$ However, to become fully competitive with fossil fuels, including the cost of energy storage, electricity from solar panels has to become even cheaper. The materials, manufacturing, and installation costs are already pushed to their lower limit, but by increasing the electricity output per panel, the price of energy can be reduced further. ${ }^{2,3}$ This requires an increase in solar cell efficiency.

Nanomaterials display exceptionally strong light-matter interactions and are therefore promising candidates to consider for the next generation of high efficiency solar cells. ${ }^{4-6}$ The close matching between the wavelength of light and the size of nanomaterials can lead to a variety of resonant effects. ${ }^{5}$ By controlling their shape and size, nanomaterials offer greatly enhanced flexibility in tuning the angle, wavelength, and rate of both absorbed and emitted photons compared to their bulk counterparts. ${ }^{7,8}$ This so-called nanophotonic engineering allows for optimizing the optical properties for application in solar cells.

Initially, most research in the field of nanophotonics for solar cells was focused on maximizing light absorption. Nanomaterials can act as optical antennas, concentrating light from an area much larger than their physical size, leading to greatly enhanced absorption. The electrical current generated by a solar cell scales with the number of absorbed photons, and the optimization of light absorption has led to short circuit currents $\left(J_{\text {sc }}\right)$ close to the theoretical maximum. ${ }^{4}$ The open circuit voltage $\left(V_{o c}\right)$ of solar cells, however, was mostly seen as a consequence of material properties, design architecture, and fabrication and was less considered as an optical problem.

It can be shown, however, that nanophotonic engineering can also improve the open circuit voltage, by optimizing light emission of the solar cell. The connection between optimizing light absorption and increasing current is very clear, but optimization of light management for high $V_{\text {oc }}$ is less intuitive. Although the importance of emission control was already recognized by Shockley and Queisser in their seminal paper on solar cell efficiency ${ }^{9}$ and later discussed in more detail several decades ago, ${ }^{10}$ practical implementation has been limited. Improvements have been made, but the achieved open circuit voltages are still further away from the theoretical limit ${ }^{4}$ than the achieved $J_{\mathrm{sc}}$. The only exception is gallium arsenide (GaAs)

Received: January 31, 2020

Revised: May 25, 2020

Accepted: May 28, 2020

Published: May 28, 2020 
solar cells, which have been studied for their outstanding optical properties for a long time.

Most early work on emission control was based on angle restriction filters that block emission into certain angles. Only recently nanophotonic strategies started to be implemented that allow for a fundamentally different approach, with the key difference being the stage at which the emission is controlled. Angle restriction filters, even if they are based on nanoscale structures like Bragg stacks or photonic crystals, ${ }^{11-13}$ interact with the light after it has been emitted. Nanophotonic engineering changes the photonic environment of the emitter and, thereby, the emission process itself. This is particularly relevant for nonideal materials, where nonradiative recombination is dominant. With angle restrictors, the light emitted at oblique angles is reflected back, which leads to an increase of photon recycling, that is, reabsorption of emitted photons. This reabsorption enhances the nonradiative recombination. In any practical application, this increase in nonradiative recombination nullifies any increase in efficiency coming from angle restriction. ${ }^{14}$ This led to the general idea that emission control only helps for materials close to the radiative limit. However, nanophotonic structures enable emission control while simultaneously reducing nonradiative recombination. ${ }^{7,8,15-17}$ In this Perspective, we will give an overview of how nanophotonic engineering can be used to increase solar cell efficiency, with a focus on increasing the open circuit voltage, by exploring the opportunities arising from lightmatter interactions at the nanoscale that are fundamentally different from any bulk phenomena.

This Perspective is structured as follows. We start with deriving the theoretical maximum efficiency for converting solar energy to electrical power. This derivation gives insight in why light emission is an important performance parameter for solar cells and shows us how an ideal solar cell should perform. From this theoretical maximally efficient solar cell we can find three aspects in which any real solar cell differs from the ideal one. These lead to three loss mechanisms that limit the efficiency of solar cells. For each of these, we will discuss nanophotonics strategies that can be applied to minimize the losses. Finally, we will present two designs for high efficiency solar cells based on the proposed techniques, and we analyze the performance modeled under realistic operation conditions. We discuss the (optical) properties required for such cells and highlight some of the most important research questions that still need to be addressed.

\section{FUNDAMENTALS OF PV}

The upper limit on the conversion efficiency of solar to electrical energy is set by the first and second law of thermodynamics. These laws have played a major role in the development of any energy technology we have today. Applying them to solar energy conversion will give insight in how we can approach the upper limit of efficiency. In the thermodynamic model, the photovoltaic (PV) system acts as a heat engine, with the sun as the hot source, the ambient as the cold source, and the light as a heat exchange medium. Optimizing a heat engine requires optimizing the heat exchange. In the case of PV, this means optimizing the light management is a way to improve efficiency. Understanding how a PV system should operate from a thermodynamics point of view gives insight in how we should manage the light with nanophotonics.
When a heat engine extracts heat from a hot source at $T_{\mathrm{H}}$, not all energy can be converted into useful work. Since work is free of entropy, the entropy from the hot source has to go somewhere else, which requires some heat to be lost toward a cold sink at $T_{\mathrm{C}}$. The maximum efficiency of this heat engine is given by the Carnot limit:

$$
\eta_{\text {Carnot }}=1-\frac{T_{\mathrm{C}}}{T_{\mathrm{H}}}
$$

With the sun at $T_{\mathrm{H}}=6000 \mathrm{~K}$ and the ambient at $T_{\mathrm{C}}=300 \mathrm{~K}$, the maximum efficiency is $95 \%$.

This limit can be reached in theory with the following requirements: the PV system consists of an infinite stack of solar cells with smoothly varying band gap from 0 to $\infty$ with full absorption and without the presence of any nonradiative recombination and etendue expansion. ${ }^{18}$ The performance of such a system can be derived, based on the fact that any material at nonzero temperature must exchange thermal radiation with the environment to be in thermal equilibrium. According to Kirchhoff's reciprocity law, absorption and emission at a given wavelength into a given angle are always coupled. ${ }^{19}$ The emission (and thus absorption) spectrum of an ideal blackbody is described by Planck's law and is a function of temperature. The incident flux of photons with energy $E$ on a solar cell coming from the sun at temperature $T_{\mathrm{S}}$ is

$$
f_{i}=\frac{1}{\exp \left(\frac{E}{k_{\mathrm{B}} T_{\mathrm{S}}}\right)-1} \frac{2 \pi E^{2}}{h^{3} c^{2}}
$$

with $h$ being Planck's constant, $q$ being the elemental charge, $k_{\mathrm{B}}$ is Boltzmann's constant, and $c$ is the speed of light. Note that this equation assumes illumination from the full hemisphere or full concentration, corresponding to no etendue expansion between emission and absorption. When a voltage builds up in a material, the emission intensity is increased. The flux of photons $f_{0}$ emitted by a solar cell at energy $E$ depends on the temperature $T_{\mathrm{C}}$ and the voltage $V$ over the cell according to

$$
f_{0}=\frac{1}{\exp \left(\frac{E-q V}{k_{\mathrm{B}} T_{\mathrm{C}}}\right)-1} \frac{2 \pi E^{2}}{h^{3} c^{2}}
$$

This equation gives the photon flux emitted by each cell with a band gap at $E$ in the infinite stack of cells. ${ }^{18}$ In open circuit, when no electrical current is extracted from the cell, the emitted and absorbed photon flux must balance to reach thermal equilibrium. The emission spectrum of the infinite stack of solar cells then equals the emission spectrum of the sun, meaning that $f_{0}=f_{i}$ at every $E$, which requires:

$$
\frac{E-q V_{\mathrm{oc}}}{k_{\mathrm{B}} T_{\mathrm{C}}}=\frac{E}{k_{\mathrm{B}} T_{\mathrm{S}}}
$$

where $V_{\mathrm{oc}}$ is the open circuit voltage in each cell. This can be rewritten to

$$
\frac{q V_{\mathrm{oc}}}{E}=1-\frac{T_{\mathrm{C}}}{T_{\mathrm{S}}}=\eta_{\text {Carnot }}
$$

which gives a direct relation between the theoretical maximum $V_{\text {oc }}$ in each cell and the Carnot limit. It shows that the energy of the photons $(E)$ is converted to energy of electrons $\left(q V_{o c}\right)$ with the Carnot efficiency. 
From this derivation we can conclude that, to optimize solar cell performance, the emission spectrum at $V_{\text {oc }}$ must approach that of the sun. In a practical solar cell, three effects cause the emission spectrum to differ from the solar spectrum and thereby reduce efficiency. First of all, a practical solar cell consists of a limited number of materials, often only one. A single band gap material has a narrow emission spectrum that peaks close to the band gap and exponentially decreases toward higher energy. The second effect is the difference between absorption and emission angles. In the derivation above, emission and absorption happen in the same solid angle. In practice, a cell receives collimated sunlight over a narrow angle, while it emits in all directions. This increase in etendue can be seen as a heat loss toward the environment or as an increase in photon entropy. Finally, any real material provides nonradiative pathways, via which absorbed photons are lost as heat toward the environment. This reduces the emission intensity compared to the incoming sunlight.

To extract power, current must be drawn. This moves the system away from the reversible Carnot limit and reduces the maximum efficiency. Excited states now do work instead of emitting light, which reduces the emission intensity. The resulting maximum power conversion efficiency is $87 \% .{ }^{18}$ It is important to make the distinction between this useful reduction in voltage and the three mechanisms mentioned above, which decrease the voltage without increasing the current.

The well-known Shockley-Queisser limit ${ }^{9}$ is also based on thermodynamics, but for a less idealized system. It includes two of the three loss mechanisms: it assumes a single stepwise band gap and takes into account the difference in solid angle between emission and absorption, but it does not include nonradiative recombination. The system is characterized by the short circuit current density $J_{\text {sc }}$ which is the same as the photogenerated current density, and the dark current density $J_{0}$, which is the recombination current density in the dark. These two determine the open circuit voltage $V_{\text {oc }}$ according to $^{20}$

$$
V_{\mathrm{oc}}=\frac{k_{\mathrm{B}} T}{q} \ln \left(\frac{J_{\mathrm{sc}}}{J_{0}}\right)
$$

With these assumptions, the efficiency as a function of band gap can be calculated, shown as the red line in Figure 1. This calculation uses the AM1.5G spectrum with 1 sun illumination. It has a maximum efficiency of $34 \%$, which is far below the limit of $87 \%$ due to the emission being narrowband and isotropic.

Any real single junction solar cell under one sun illumination performs below the SQ limit due to nonradiative recombination of excited electrons. By analyzing the performance of a record efficiency silicon solar cell, we can quantify how much each of the three loss mechanisms contributes to a reduction in current and voltage. For this analysis we assume the solar cell has a stepwise band gap of $1.1 \mathrm{eV}$. The amount of nonradiative recombination is given by the photoluminescence quantum yield (PLQY), which is the ratio of radiative recombination to the total (radiative plus nonradiative) recombination. We use a PLQY of $1 \%$, which is the typical value for state-of-the-art silicon solar cells. ${ }^{21,22}$ With these assumptions, the modeled cell has an efficiency of $28 \%$ under the AM1.5G spectrum. The actual record lies at $26.7 \%,{ }^{23}$ meaning that other nonidealities that are not considered in this model account for only $1.3 \%$.

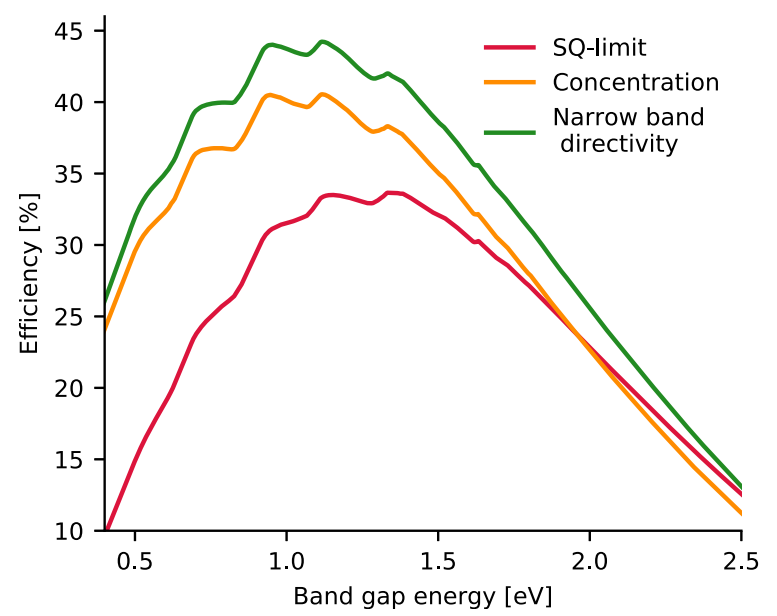

Figure 1. Maximum achievable efficiency as a function of band gap energy based on the AM1.5 spectrum for three models: the SQ limit (blue), maximum concentration of 46200 (orange), and maximum directivity of 46200 over a narrow bandwidth optimized at each band gap (green). The latter one also captures the diffuse part of the light efficiently.

These are, for example, no ideal stepwise absorption onset and imperfections at the contacts. The remaining difference between the record efficiency and the maximum achievable efficiency of $87 \%$, can be split up in different contributions, as shown in Figure 2. The fraction of photons that are not

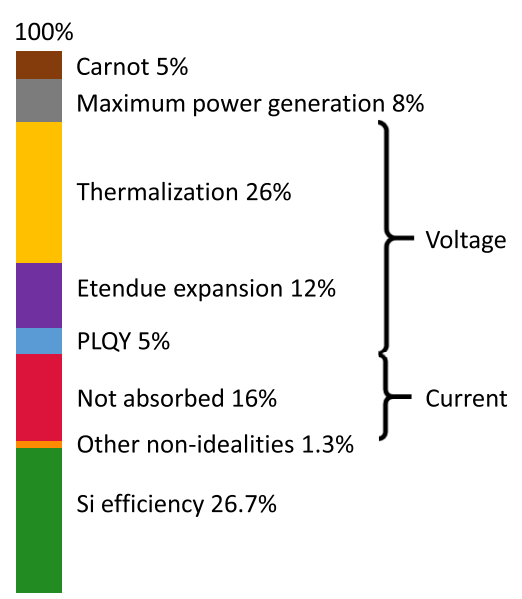

Figure 2. Contribution of different loss mechanisms to the total reduction in power conversion efficiency compared to the thermodynamic limit. Most losses (43\%) are caused by a reduction in voltage.

absorbed contain only $19 \%$ of the incoming energy. Considering that the conversion efficiency at maximum power generation is $87 \%$, the energy loss due to nonabsorbed photons, and thus, loss in current is $16 \%$. The remaining $43 \%$ of efficiency loss compared to the maximum power conversion efficiency is due to reductions in voltage originating from increases in $J_{0}: 5 \%$ efficiency loss due to the nonradiative recombination, $12 \%$ loss due to isotropic emission, and $26 \%$ due to thermalization of high energy electrons. In the following sections we will discuss each of the loss mechanisms in more detail and provide nanophotonic concepts that can help to overcome these losses. 


\section{IMPROVING LIGHT EMISSION}

The reduction in emission intensity due to nonradiative recombination is the first loss mechanism that we will discuss. Any nonradiative recombination increases the saturation current density $J_{0}$ and thereby reduces the voltage compared to the radiative limit $V_{\text {oc,rad }}$ according to ${ }^{20}$

$$
V_{\mathrm{oc}}=V_{\mathrm{oc}, \mathrm{rad}}+\frac{k_{\mathrm{B}} T}{q} \ln (P L Q Y)
$$

The radiative limit is very similar to the SQ limit, but takes into account the realistic, nonstepwise absorption spectrum. A high performance solar cell should have good absorption anywhere above the band gap and all of the discussion below assumes that good absorption is achieved. The radiative limit is not approached by reducing $J_{0}$ when $J_{\mathrm{sc}}$ is not maintained, because efficiency changes only logarithmically with $J_{0}$ (eq 6), while it changes linearly with $J_{\text {sc }}$.

In approaching the radiative limit, PLQY is the key performance parameter. It is the rate of externally emitted photons divided by the total recombination rate and is determined by two parameters. One is the internal luminescence efficiency $Q_{i}^{\text {lum }}$, which depends on the radiative and nonradiative rates inside the material. The second is the escape probability $p_{\mathrm{e}}$ : the probability that an internally generated photon makes it to the far field, outside the material. Otherwise, it is reabsorbed with probability $p_{\mathrm{r}}$ or parasitically absorbed with probability $p_{\mathrm{a}}$, such that $p_{\mathrm{e}}+p_{\mathrm{a}}+p_{\mathrm{r}}$ $=1$. The PLQY expressed in these terms is given by ${ }^{20}$

$$
P L Q Y=\frac{Q_{\mathrm{i}}^{\operatorname{lum}_{\mathrm{e}}}}{1-Q_{\mathrm{i}}^{\text {lum }} p_{\mathrm{r}}}
$$

To approach the radiative limit, we have to maximize $Q_{i}^{\text {lum }}$ and $p_{\mathrm{e}}$. The internal luminescence efficiency $Q_{i}^{\text {lum }}$ is increased by decreasing the nonradiative recombination rate or by increasing the radiative rate. The probability $p_{\mathrm{e}}$ is increased by improving light outcoupling. Reabsorption in the active material is preferable over parasitic absorption in other materials, so increasing $p_{\mathrm{r}}$ can increase solar cell performance. Once $p_{\mathrm{a}}$ is minimized, $p_{\mathrm{e}}$ should be increased in order to approach the radiative limit.

The nonradiative recombination rate constant is set by the material quality and scales with the density of defects. Nanomaterials can show higher material quality, because they are more easily grown as single crystals. Also lattice mismatch with the substrate is more easily accommodated, allowing for a wider range of materials to be combined. The large surface-to-volume ratio was for a long time considered to be the main drawback of nanomaterials, because many trap states are formed at the surface. ${ }^{24}$ However, great progress in surface passivation techniques has led to nanostructures with low nonradiative recombination rates. For example, silicon nanostructures (black silicon) passivated by $\mathrm{Al}_{2} \mathrm{O}_{3}{ }^{25}$ or $\mathrm{SiO}_{2}{ }^{26}$ InP nanowires passivated by $\mathrm{PO}_{x}{ }^{27}$ and halide perovskite nanowires passivated by porous aluminum oxide templates ${ }^{28}$ have all enabled PLQY values comparable to state-of-the-art bulk systems.

Both the radiative recombination rate and light outcoupling can be tuned with nanophotonic engineering. When comparing a planar device to a nanostructured device, many parameters are changed at the same time and it is difficult, if not impossible, to fully separate the different contributions to the final performance. A detailed (mathematical) analysis is beyond the scope of this paper. We will point out some of the effects that play a role.

The key parameter to which many of the optical properties of nanostructures relate is the enhancement in light absorption. Initially, this was mainly considered as a way to increase $J_{\text {sc }}$. However, it can also improve $V_{\text {oc }}$ by increasing PLQY, as given in eq 7. Absorption enhancement per unit volume leads to a higher density of excited charge carriers inside the material, that is, a higher injection level. The radiative recombination rate scales with the carrier concentration squared, while the trap state assisted nonradiative recombination rate scales only linearly with carrier concentration. ${ }^{29}$ The nonradiative Auger recombination scales with carrier concentration cubed and starts to dominate at high injection levels. ${ }^{29}$ Therefore, $Q_{i}^{\text {lum }}$ usually has a peak at finite carrier concentration; with higher carrier concentration leading to a loss in $Q_{i}^{\text {lum }}$ because of Auger recombination and lower carrier densities leading to a loss because of enhanced trap state assisted nonradiative recombination. $^{29}$ Intelligent choice of doping concentrations and absorber layer properties may allow the device to be designed such that the optimum injection level is close to one sun conditions. The effect of enhanced $Q_{i}^{\text {lum }}$ at higher injection levels can also be understood from reciprocity: a higher absorption rate means also a higher emission rate; for the same nonradiative rate this gives an increase in $Q_{i}^{\text {lum }}$.

Changes in light absorption and, thus, light incoupling, will also influence the light outcoupling and, thereby, $p_{\mathrm{e}}$. Intuitively, one can understand that when less absorbing material is needed, $p_{\mathrm{e}}$ will be larger in a nanostructured device: emitted light has to travel a shorter distance through the material before it reaches an interface where it can escape or, again, the reciprocity argument can be used: stronger coupling to incoming light means stronger coupling to outgoing modes, which increases $p_{\mathrm{e}}$.

The changes in $Q_{i}^{\text {lum }}$ (via enhanced internal radiative rate) and $p_{\mathrm{e}}$ (via enhanced external radiative rate) are related to the same optical effects. Nanostructures can enhance the local density of optical states (LDOS), and they can enhance coupling to specific modes, which is equivalent to locally enhancing the field of incoming waves. Since these effects are coupled and influence both $Q_{i}^{\text {lum }}$ and $p_{\mathrm{e}}$, these parameters can not be tuned independently. In addition to that, the absorption and emission spectra will be altered by changes in LDOS and electric field strength. Often, the consequential changes in $V_{\mathrm{oc}}$ are attributed to different parameters in an additive manner, but it has already been shown that in this additive form the different components are not independent. ${ }^{30}$ By combining the additive terms in one equation (eq 27 in ref 30), this expression is claimed to contain only independent parameters. However, when considering nanostructures, several terms in this expression are still coupled via the LDOS and field enhancement. A general statement on how these effects change $V_{\text {oc }}$ can therefore not be made. However, attempts to attribute changes in $V_{\text {oc }}$ to specific parameters has led to some confusion, some of which we will try to resolve here.

In the first place, there is experimental proof of strongly increased $V_{\mathrm{oc}}$ and PLQY in nanostructured materials. By comparing a planar InP device to one with nanowires of the same material, an increase in $V_{\text {oc }}$ of $70 \mathrm{mV}$ was found due to a combination of enhanced absorption, increased $Q_{i}^{\text {lum }}$, and increased $p_{\mathrm{e}}{ }^{15}$ Light outcoupling enhancement via nanotexturing has also been used to improve methylammonium 
lead halide perovskite thin film solar cells. For the iodide perovskite, the PLQY was increased by more than a factor of 5, by going from thin film deposition on a smooth glass substrate to a nanotextured substrate. ${ }^{31}$ Also, without fully quantifying and separating the contributions of the physical processes underlying these effects, it should be clear that nanostructures can improve the optical properties of a material.

One source of confusion is the fact that changing the optical properties of a material can also change the emission and absorption spectrum. This might lead to an effective increase or decrease in band gap, resulting in a change in $V_{\text {oc,rad. }}$. When only looking at the resulting voltage, one can come to the conclusion that $p_{\mathrm{e}}$ should be minimized, because this can lead to an effective increase in band gap and, therefore, in $V_{\text {oc. }}{ }^{32}$ However, this is only moving along the band gap axis in Figure 1 , without actually getting closer to the radiative or SQ limit, as shown by the red arrow in Figure 3. In the presence of

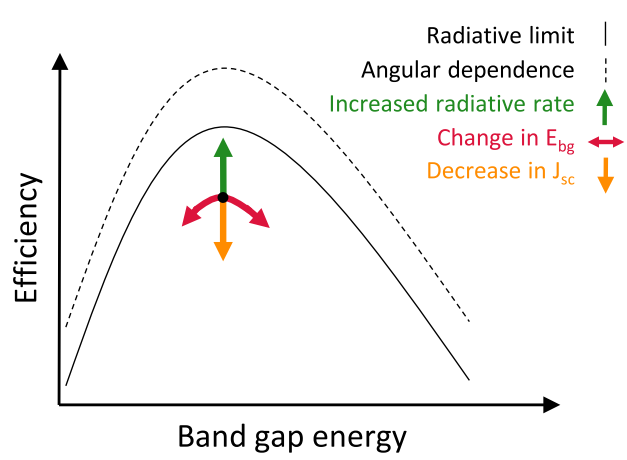

Figure 3. Nanostructures can affect the efficiency and $V_{\text {oc }}$ in several ways, which occasionally leads to some confusion. Four effects can be distinguished: by enhancing the radiative rate, the PLQY can be increased, approaching the radiative limit (green arrow); the effective band gap can be changed (red arrow); a resonance at the band gap effectively decreases $J_{\text {sc }}$ and $V_{\text {oc,rad }}$ (orange arrow); or the whole radiative limit can be increased by restricting the cone of emission (dotted line).

nonradiative recombination, increasing $p_{\mathrm{e}}$ will lead to an increase in PLQY (green arrow in Figure 3). Even though the absolute $V_{\text {oc }}$ may decrease due to a lower effective band gap, the radiative limit will be more closely approached. Intuitively, this can be well-understood from the fact that any reabsorption of a photon (due to a low $p_{\mathrm{e}}$ ) provides another chance for nonradiative recombination, which reduces the PLQY. Therefore, $p_{\mathrm{e}}$ should always be maximized for approaching the radiative limit as long as absorption is not compromised.

Another source of confusion arises when looking at individual nanoparticles. Instead of an absorption spectrum, they are typically characterized by their absorption cross section $\sigma_{\mathrm{abs}}$ as a function of wavelength, defined as the effective area over which the particle absorbs light. The absorption cross section can be substantially larger than the geometrical size of the particle, which makes the standard definitions for solar cell performance not applicable. ${ }^{33}$ In individual nanoparticles, $V_{\text {oc,rad }}$ and the radiative recombination rate can be changed over a very large range, which complicates fair comparisons between different systems. In Figure 3, we show schematically the different situations that can be encountered. When $\sigma_{\mathrm{abs}}$ is increased equally for all wavelengths above the band gap, the radiative rate is increased. This brings the system closer to the radiative limit, as indicated by the green arrow. As long as $\sigma_{\mathrm{abs}}$ is isotropic, the efficiency can not increase above the radiative limit, because $J_{0, \text { rad }}$ (the radiative saturation current density) and $J_{\text {sc }}$ are increased by the same amount. To surpass the radiative limit, the expansion of etendue has to be reduced, which is the topic of the next section. This requires an angledependent $\sigma_{\text {abs }}{ }^{33}$ and as a consequence the whole radiative limit moves to higher efficiency for all band gaps (dotted line). If $\sigma_{\mathrm{abs}}$ is increased more for some wavelengths than for others, the effective optical band gap of the material is changed and one moves along the band gap axis, indicated by the red arrow. Depending on whether the starting point is below or above the optimum band gap, this increases or decreases efficiency. When an individual nanoparticle has a strong resonance at the band gap, $V_{\text {oc,rad }}$ can actually be decreased significantly. The resonance strongly enhances emission and absorption at the band gap only, by which $J_{0}$ is increased much more than $J_{\text {sc }}{ }^{33,34}$ following the orange arrow. In a full scale solar cell, this would be equivalent to a device with full absorption at the band gap, but much lower absorption at higher energy. Since a good performing solar cell should have full absorption anywhere above the band gap, larger absorption rates at the band gap than at higher energy are not desirable. By designing a structure with a both angular and wavelength-dependent absorption cross section, potentially very high efficiencies can be achieved, as shown by the green curve in Figure 1. This will be discussed in more detail in the next section.

Throughout this section, we have used the PLQY as a measure of solar cell voltage, relying on the reciprocity relation between light absorption and light emission. The critical reader might have noticed that this is technically incorrect, because the reciprocity relation links PLQY to the quasi-Fermi level splitting, while solar cell voltage is connected to the electroluminescence (LED) quantum efficiency. The PLQY and LED quantum efficiency are equivalent when assuming perfect contacts and infinite carrier mobility, but can be different for real materials. ${ }^{35}$ However, in mature semiconductor systems, carrier extraction is close to perfect, and for most materials, except for organics, the mobility is sufficiently high at one sun fluxes. In this case, transport losses do not play a significant role, and the optically and electrically measured quantum efficiency are almost the same. ${ }^{35-37}$ PLQY is the upper bound and is the one we influence with nanophotonics. We do not focus on carrier transport or carrier injection in this Perspective and, therefore, use PLQY as a characteristic parameter. Experimentally, PLQY is also often more useful since it can be measured at every step of the solar cell fabrication, while the electroluminescence requires a complete device.

\section{MATCHING THE ANGLES}

The second loss mechanism that we discuss is the mismatch between absorption and emission angles. This leads to an increase in photon entropy between the incoming and emitted light. Since the conventional SQ limit assumes isotropic emission, this limit can be exceeded by reducing the cone of emission. This leads to a decrease in the radiative recombination $J_{0, \text { rad }}$ by which $V_{\text {oc,rad }}$ and, thus, the efficiency are increased. Emission and absorption are coupled via Kirchhoff's law, so emission into the cone of the sun can not be reduced without sacrificing $J_{s c}$. The sunlight comes from a cone with a $0.5^{\circ}$ half angle, which allows for a maximal reduction of $J_{0}$ by a factor 46200 compared to emission into 
the full hemisphere. This corresponds to a maximum increase in voltage of $278 \mathrm{mV}$.

The most conventional way to reduce the loss due to etendue expansion is by using a concentrating lens. The light that comes directly from the sun is focused onto the cell, while the thermal radiation that comes from all angles is not focused on the cell. Or, equivalently, the lens focuses the emission from the cell toward the sun. The resulting efficiency of the SQ limit with $46200 \times$ concentrated AM1.5 spectrum is shown in Figure 1 , orange line. The maximum achievable efficiency is over $40 \%$. However, in a typical terrestrial situation, part of the sunlight is scattered by clouds and surroundings. This light is not focused on the cell and as a consequence the absorption of this diffuse light decreases by the concentration factor. In many terrestrial applications, this decreases $J_{\mathrm{sc}}$ more than it increases $V_{\mathrm{oc}}$ and the efficiency decreases.

To see the consequences of this effect, we analyzed the impact of diffuse sunlight on conventional concentrating systems. We derived an expression for the relation between efficiency, concentration factor and fraction of direct sunlight. For a concentration factor $X$, both $J_{0}$ and the absorption of diffuse sunlight are reduced by a factor $X$. Plugging this into the equations for $J_{\mathrm{sc}}$ and $V_{\mathrm{oc}}$ and simplifying the expression by neglecting small terms, it can be shown that efficiency approximately scales with concentration factor and fraction of direct sunlight as

$$
\eta \propto J_{\mathrm{sc}} f_{\mathrm{dir}}\left[\ln \left(\frac{J_{\mathrm{sc}}}{J_{0}}\right)+\ln \left(f_{\mathrm{dir}} X\right)+\frac{1}{X} \frac{1-f_{\mathrm{dir}}}{f_{\text {dir }}} \ln \left(\frac{J_{\mathrm{sc}}}{J_{0}}\right)\right]
$$

with $J_{\mathrm{sc}}$ and $J_{0}$ being the short circuit and dark current for the cell without a concentrating lens, respectively, and $f_{\text {dir }}$ is the fraction of direct sunlight. The derivation can be found in the supporting information. This equation shows that the concentration factor has both a positive $(\ln (X)$ term) and a negative $(1 / X$ term $)$ effect on efficiency, which originate from the increase in $V_{\mathrm{oc}}$ and the decrease in $J_{\mathrm{sc}}$, respectively.

We used this expression to calculate the efficiency as a function of concentration factor for a silicon solar cell, ignoring Auger effects. We used a band gap of $1.1 \mathrm{eV}$ and the AM1.5 spectrum, which contains $90 \%$ direct sunlight, and a modified version of this spectrum with $85 \%$ direct sunlight. Figure 4 shows how, at low concentration, the reduction in $J_{s c}$ is dominant and the efficiency is decreased. Only above a certain

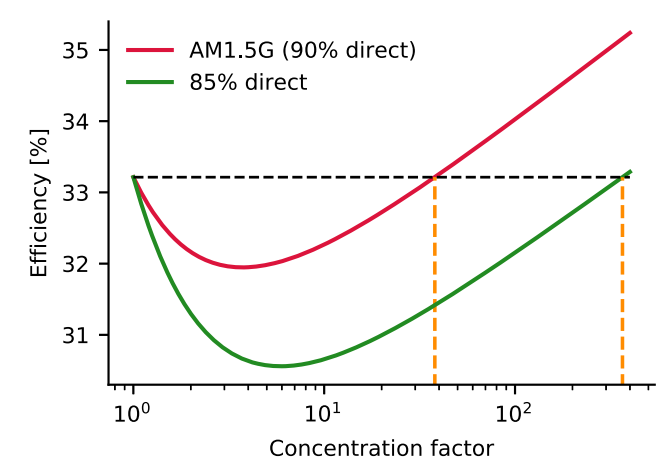

Figure 4. Efficiency as a function of concentration factor at a band gap of 1.11 for different fractions of direct sunlight, calculated with eq 9. The black line indicates the efficiency at 1 sun and the orange lines indicate the break even points above which the efficiency is increased due to the concentration. threshold value for $X_{\text {thr }}$, concentration is beneficial. The lower the fraction of direct sunlight, the higher $X_{\mathrm{thr}}$ becomes. $X_{\mathrm{thr}}$ rapidly increases by going from $90 \%$ to $85 \%$ direct sunlight; it increases from 40 to almost 400 . Vice versa, the lower the concentration factor, the more direct sunlight is needed. The minimum required fraction of direct sunlight for a given $X$ can be derived from eq 9 and reads

$$
f_{\text {dir }}=\left[1+\frac{1}{\ln \left(J_{\mathrm{sc}} / J_{0}\right)} \frac{X \ln (X)}{X-1}\right]^{-1}
$$

For the AM1.5 global spectrum, a band gap of $1.1 \mathrm{eV}$ and maximum concentration (46200×), at least $76 \%$ of the sunlight must be direct. For a more practically achievable concentration of $400 \times$, the break-even point lies just below $85 \%$ direct sunlight. In many places on earth this value is not reached most of the time. In that case, adding a concentrator will make the system perform worse. To get a feeling for the numbers: in The Netherlands, the fraction of energy from direct sunlight is on average $36 \%$, in Denver (Colorado, U.S.) this is $76 \%$ and in the Sahara this is $80 \% .^{38}$ The AM1.5G spectrum assumes a clear sky but still contains $10 \%$ diffuse sunlight due to the presence of the atmosphere.

Instead of expanding the angular range of incident sunlight to match the angle of emitted light (as with concentrating lenses described above), it is also possible to reduce the emission angle of the solar cell toward that of the sun. This is done with angle restrictive filters, that block emission and absorption into oblique angles. This concept was described more than two decades ago ${ }^{10}$ and has been studied in order to find upper thermodynamic limits for PV systems since then. ${ }^{30,39}$ When acting over the full solar spectrum, angle restrictive mirrors are mathematically equivalent to concentrating lenses and they can be described with an effective concentration factor. However, since emission occurs over a narrow bandwidth, the angle restrictive mirrors can be made wavelength-selective. This way, the problem with diffuse sunlight can be circumvented: if the mirrors only block light at the emission wavelength, while they are transparent at higher energy, $J_{0}$ is decreased while maintaining high $J_{\mathrm{sc}}$. In practice, however, PV systems with angle-selective mirrors suffer from the decreased escape probability, which reduces the PLQY, as discussed in the previous section (eq 8), as a consequence of photon recycling. The resulting increase in $V_{\text {oc }}$ for an effective concentration factor $X$ and initial value of $\mathrm{PLQY}$ is given by $\left(k_{\mathrm{B}} T / q\right) \ln \{X /[X-(X-1) \mathrm{PLQY}]\}$, which approaches zero as the initial PLQY goes to zero. ${ }^{21}$ This approach was tried experimentally using a high quality GaAs solar cell. Despite the high PLQY in GaAs, the increase in $V_{\text {oc }}$ was only a few $\mathrm{mV}$ due to the decrease in escape probability. ${ }^{40}$

As mentioned above, concentration and angle restriction are mathematically the same in the radiative limit and without considering diffuse sunlight. When you look at the combined system of solar cell plus concentrating lens or angle restrictor from the outside, both look the same: only incoming light at normal incidence is absorbed, and only collimated light comes out. Both techniques have their limitations and, by combining the two losses, can be minimized. ${ }^{11,41}$ Concentrating lenses always act over the full spectrum, which leads to significant losses in any realistic operating conditions with diffuse sunlight. However, they do have the advantage of having good light outcoupling, which makes them also applicable to materials with a low PLQY like silicon. Angle restrictors allow 
for wavelength selectivity, but they work based on reduced light outcoupling. In some cases this can be beneficial, because this light trapping increases the absorption close to the band gap, increasing $J_{\mathrm{sc}}$ for a given thickness. ${ }^{42}$ However, this comes at the cost of increased nonradiative recombination, meaning that the efficiency will always be below the radiative limit for nonideal materials. Since most work on controlling the emission angles has been focused on angle restricting filters on top of the solar cell, which enhance photon recycling and reduce escape probability, this has led to the misconception that any type of angle restriction only works for solar cells close the radiative limit. With nanophotonic structures, this does not have to be the case: they change the emission event itself, instead of interacting with the light after it is emitted. Just like the efficiency of silicon cells can be increased with concentrating lenses, which effectively also change the angular profile of the emission, nanophotonic structures have the potential to improve efficiency also for materials with low radiative efficiency. ${ }^{8}$

Nanophotonic structures can combine the best of both worlds: maintaining high light-outcoupling as with concentrating lenses while providing wavelength selectivity comparable to angle restriction filters. This requires directive nanoparticles: particles that emit and absorb more in one direction than in all others. This is described by the so-called directivity, as used in antenna theory. It is defined as the maximum power absorbed or emitted into a certain direction divided by the average power in all directions. The particles must be directive over a narrow bandwidth around the emission wavelength and absorb high energy light from all directions. This gives both directive light emission (i.e., reduced emission loss) and good absorption of diffuse sunlight while maintaining or potentially increasing the escape probability. The resulting efficiency of such a system, with the bandwidth of directivity optimized at each band gap, is shown in Figure 1, green line.

To achieve these efficiencies, one must have accurate control over the light emission. In the field of nanophotonic engineering, several methods have been developed for controlling the emission at the nanoscale. Especially in the field of single photon emitters, there is a large demand for increasing the brightness, enhancing the Purcell factor, and controlling the directivity. ${ }^{7}$ A frequently studied system is the Yagi-Uda antenna. On a macroscopic scale, this is a wellknown antenna design used for radio frequencies. By scaling it down to the nm size, light emission of a single quantum dot can be controlled. Using five gold elements, a forward-tobackward ratio of the emission of $4.7 \mathrm{~dB}$ has been experimentally achieved. ${ }^{43}$ Another well-known geometry is the Bull's eye antenna, which consists of concentric rings or grooves around the emitter(s). With shallow grooves in a gold film, a directivity of $7.5 \mathrm{~dB}$ was measured from an ensemble of particles. ${ }^{44}$ Coupling emitters to plasmonic resonances in an array of aluminum nanoantennas gave a more than 60 -fold directional enhancement for the purpose of efficient lightemitting diodes. ${ }^{45}$ By introducing spatial variations in an array of nanoantennas, wavefront shaping with subwavelength resolution can be achieved, which is being used for the development of flat optics or metalenses. ${ }^{46}$

All of the above-mentioned systems rely on plasmonic nanostructures. The main disadvantage of plasmonic structures is the parasitic absorption in the metals. This realization has led to a shift away from lossy metallic (plasmonic) resonators toward dielectric systems. Here, many of the nanophotonic advantages are still present, but without the parasitic absorption. ${ }^{47}$ For example, a hybrid system of a titania Bull's eye structure on a silver film was realized, which gave emission into two lobes with full-width-half-max of $3^{\circ} .^{48}$ An all-dielectric Yagi-Uda nanoantenna was fabricated from silicon spheres, reaching a maximum directivity above 7 in the microwave range. ${ }^{49}$ Also, in the field of metalenses, the low efficiency associated with the dissipative losses in metals has led to the development of all-dielectric metasurfaces, which show good potential for replacing their plasmonic counterparts. ${ }^{50}$

For the performance of solar cells, parasitic absorption is detrimental for both current and voltage. Initially, work has been done on plasmonic structures for solar cells, mostly to enhance absorption in the solar cell material. ${ }^{51}$ Since it became clear that the parasitic absorption in the metals will limit the achievable efficiencies, the attention has now shifted to dielectric nanostructures. ${ }^{5}$

Of course, the dielectric materials have their own possibilities and challenges. An advantage is that dielectric nanoparticles can support both electric and magnetic resonances simultaneously, which can be controlled independently. ${ }^{47}$ On the other hand, these resonances are usually less localized than in metals, leading to more broadband response and lower directivity. Fabrication imperfections are challenging in any nanoscale structure and cause suboptimal geometries and rough surfaces. Insufficient surface passivation has further decreased the performance of dielectric nanophotonic structures so far. Both theoretical and experimental work is to be done on improving light management with dielectric nanostructures and developing pathways to large-scale applications.

A specific example of the kind of design requirements is the angular and wavelength dependent absorption and emission profile needed for the reduction in etendue. For a material with a band gap at $860 \mathrm{~nm}$ (e.g., GaAs), the ideal absorption cross section as a function of angle and wavelength is shown schematically in Figure 5. The structure must have high absorption at normal incidence to collect the direct sunlight efficiently and high absorption from all directions at high energy to maximize absorption of diffuse sunlight. Close to the

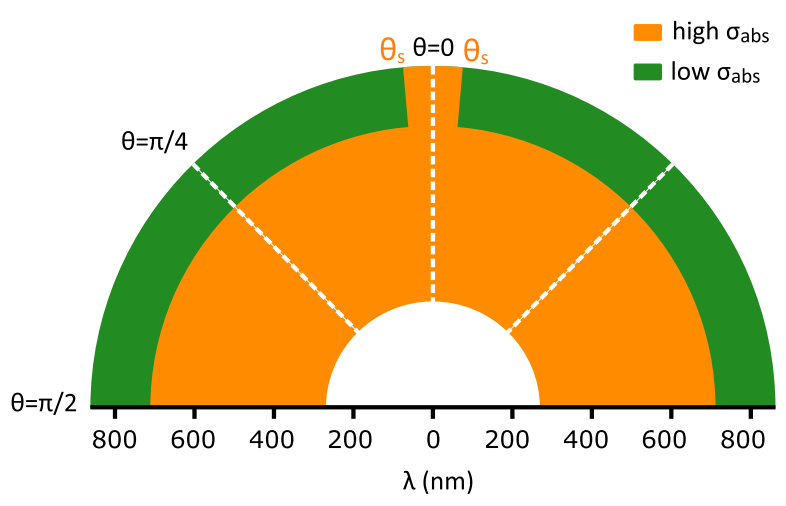

Figure 5. Schematic illustration of how the absorption cross section $\left(\sigma_{\text {abs }}\right)$ of a nanostructure should vary with wavelength and angle of the incoming light in order to achieve the narrow band directivity. Around the band gap, the nanoparticle should be directive, such that it emits light in the direction of the sun. This requires higher absorption for normal incidence than for oblique angles. At energies further above the bandgap, it should absorb light from all directions to also capture diffuse sunlight. 
band gap, away from normal incidence the absorption should be low, which makes the emission directional toward the sun. Radiative recombination should be enhanced, while parasitic absorption must be avoided.

To realize such particles, morphologies have to be found and fabricated that lead to anisotropic absorption cross sections. Finding the optimal shape for highly directive particles is a nontrivial nanophotonics problem. Either the absorber material itself can be made into a directive structure, or nanoantennas of dielectric material can be placed around or on top of the semiconductor. The directivity of GaAs nanowires has been increased from 2 to 20 by placing a "nanolens" on top of the wire. The nanophotonic structure of the lens was designed through an evolutionary algorithm and fabricated using twophoton lithography. ${ }^{52}$ For a future large-scale application, faster fabrication methods are needed based on chemical growth or self-assembly. A good candidate might be the shapepreserving transformation of carbonate minerals into semiconductors. Carbonate minerals can mineralize into threedimensional shapes in a well-controlled manner. Subsequently, the structure can be transformed into, for example, methylammonium lead halide perovskite, which is a good solar cell material. ${ }^{53}$

Potentially, high directivities can be reached by combining different effects. For example, it may be possible to place the directive nanolenses in an array that provides additional directivity. Another option is to choose specific emitter materials with intrinsic directional emission due to preferred dipole orientation ${ }^{54,55}$ and combining this with nanolenses or array effects or both. To achieve the wavelength-selective directivity, different materials or molecules with specific dipole orientation must be combined and coupled.

\section{MATCHING THE SPECTRUM}

The biggest loss mechanism that applies to all single junction solar cells is the mismatch in absorption and emission spectrum. For the typical silicon solar cell, as analyzed in Figure 2, this contributes to an absolute loss of $42 \%$ in efficiency. All photons below the band gap are not absorbed, which accounts for $16 \%$ of the loss, and all excess energy of above-band gap photons is dissipated as heat, thereby losing the remaining $26 \%$ of efficiency. There are several techniques available to reduce these losses. In this section we will discuss the ones that can benefit from nanophotonics.

A luminescent solar concentrator (LSC) consists of a plate or sheet of transparent material with embedded luminescent particles. These particles absorb the sunlight and subsequently emit light, which is then waveguided through the plate. Small solar cells connected to the waveguide absorb the light and convert it to electricity. Although this system has the name concentrator, it can not increase the efficiency above the SQ limit, like the concentrating systems discussed previously. This can be understood from the fact that the thermal emission to the environment is not directed toward the sun, and thus, the entropy loss remains. However, LSCs have several advantages, both economical and physical, and nanophotonics can help to improve their performance.

The economical benefit of using an LSC is that the transparent plate with luminescent particles can be cheap and easy to fabricate: existing cover glasses or polymer coatings used in the solar cell industry can already fulfill the role of a waveguide. The actual solar cells to which the light is guided are very small compared to the collection area. This small size makes expensive, high efficiency solar cells affordable. LSCs can potentially create high efficiency devices, because they can be used in tandem configurations for better capturing of the full solar spectrum. Conventional multijunction solar cells are complex and expensive to fabricate, because many additional layers are required to accommodate the lattice mismatch, charge transport and surface passivation. The cells are usually connected in series, which limits the current to the lowest current in one of the cells. Stacking LSCs with different band gaps is less complex, and the cells do not need to be current matched. This makes the tandem less sensitive to temperature and spectral fluctuations and easier to fabricate. Promising results have been found in both theoretical ${ }^{56}$ and experimental $^{57}$ work on the performance of LSC-tandem configurations.

Nanophotonics engineering is already being used for optimizing the absorption and emission spectra of the luminescent particles in LSCs. With Stokes shift engineering, that is, the shift between absorption and the emission spectrum, reabsorption in the waveguide can be reduced. By properly matching the emission to the absorption of the solar cells, efficiency can be increased. In a tandem configuration, the band gaps of the different layers can be accurately tuned for optimal performance by using quantum confinement or resonances to change the effective band gap of the material. However, the main challenge for making high efficiency LSCs is to achieve efficient guiding of the emitted light toward the solar cells. Many photons are often lost along the way due to nonradiative recombination in the particles or because they are emitted into the escape cone of the waveguide. The light that is coupled to the waveguide is often reabsorbed many times along its way. Each reabsorption event leads to another chance of nonradiative recombination or emission into the escape cone.

With nanophotonic engineering, particles can be designed that minimize the losses in the waveguide. By creating directive emission into the plane of the waveguide, the fraction of light emitted into the escape cone can be reduced significantly. It has been proposed to use, for example, photonic structures to enhance in-plane emission and reduce escape cone losses. ${ }^{58}$ By creating aligned directive emission into only one direction, also the chance of reabsorption can be reduced: the structures emit light mostly in one direction (say toward the right), but due to reciprocity, they mostly absorb light traveling to the left. This leads to a structure in which light can travel only in one direction. The structure requires a lower directivity than the nanolenses in the previous section: as long as the light is emitted into the cone of total internal reflection of the waveguide, the photons do not escape. The required absorption cross section as a function of angle and wavelength is schematically shown in Figure 6. Again, at short wavelengths (high energy), the absorption should be high from all directions, to maximize absorption of sunlight, and the particles should be only directive over their emission spectrum.

Another system that reduces the spectral mismatch and that can benefit from nanophotonic engineering, is a photon multiplier. In this concept, high energy photons are converted to two low energy photons. This process takes place in the socalled down converter layer on top of a solar cell, from which the photons have to be emitted toward the solar cell. This down conversion can be realized with singlet fission ${ }^{59}$ or multiple exciton generation. ${ }^{60-62}$ The efficiency of this type of device can be increased by coupling the down converter to 


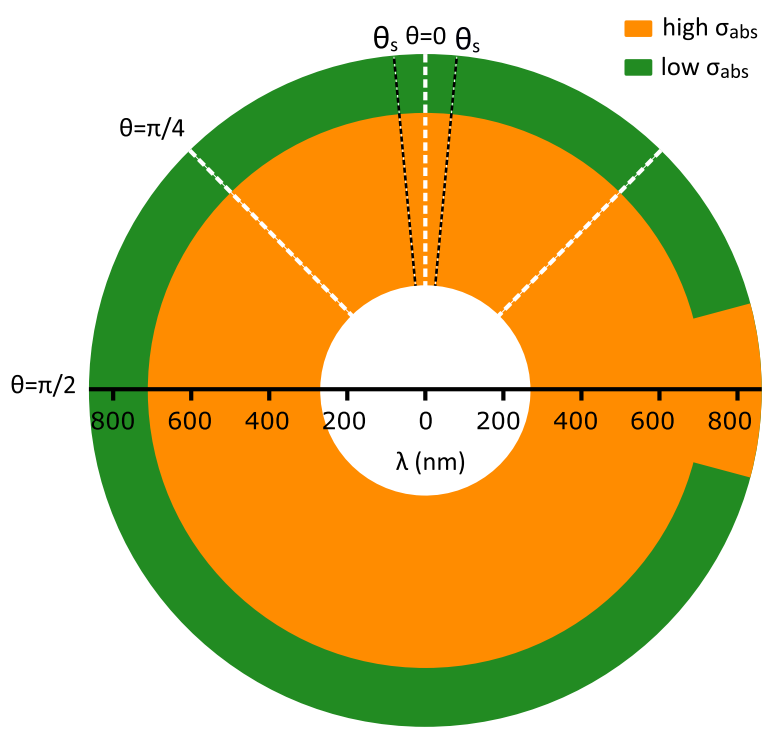

Figure 6. Schematic illustration of how the absorption cross section $\left(\sigma_{\mathrm{abs}}\right)$ of a nanostructure should vary with wavelength and angle of the incoming light for the emitters in the LSC waveguide. The emission around the band gap should occur primarily within the cone of total internal reflection, which requires low $\sigma_{\mathrm{abs}}$ in all other directions. At energies higher above the band gap $\sigma_{\mathrm{abs}}$ should be large to absorb the incoming sunlight from all directions.

directive emitters. This will ensure that the down-converted light is emitted toward the solar cell.

Finally, nanostructures can form an alternative configuration for tandem or multijunction solar cells. Instead of placing layers that absorb different parts of the solar spectrum on top of each other, spectral splitting can be achieved by placing nanostructures next to each other. Thanks to the enhanced absorption cross section of nanowires, adjacent wires with different band gap can collect light from an overlapping area. Spectral splitting can be achieved by making high band gap nanowires taller, such that the high energy photons are absorbed before they reach the lower band gap material wires ${ }^{63}$ or by making use of structures with different resonance frequencies, which cause an enhancement in absorption crosssection at specific frequencies. ${ }^{64}$

\section{FUTURE SOLAR CELLS}

In this section we propose two concepts for high efficiency solar cell designs based on the approaches discussed in the previous sections. In the first design, a high efficiency is reached by reducing the cone of emission while maintaining high $J_{\mathrm{sc}}$ and $p_{\mathrm{e}}$ by using narrow-band directive nanostructures. The second design combines existing bifacial silicon solar cells with a luminescent solar concentrator, which leads to a low cost, high efficiency tandem device. The performance of each design is evaluated in realistic operating conditions using measured solar spectra and compared to a more conventional design without nanophotonics.

Narrow Band Directivity Nanolenses. The first concept aims at a reduction in emission cone, high light outcoupling, and good absorption of diffuse sunlight. This is achieved with nanostructures that emit the light into one specific direction rather than using structures that redirect the light after emission, such that directivity does not come at the cost of light outcoupling. The constituent nanostructures should have an absorption cross section varying with incidence angle and wavelength, as shown in Figure 5. The optimal band gap for this concept is $1.1 \mathrm{eV}$, as can be seen in Figure 1. We assume a high quality solar cell material in combination with good light outcoupling to give a PLQY of $85 \%$. This could be achieved with, for example, an alloy of III-V or halide perovskite materials, which provide a tunable band gap and high PLQY. For comparison with state of the art concentrators, we propose a directivity of 400 , similar to existing record concentrating solar cells. The optimum bandwidth of the directivity was found to be $0.22 \mathrm{eV}$ based on simulations with the measured solar spectra. This design is compared with solar cells of the same material (band gap of $1.1 \mathrm{eV}$ and $85 \%$ PLQY), without concentration and with macroscopic concentration over the full bandwidth.

With these parameters, the performance of the cells can be calculated for any given solar spectrum. The spectra were taken from publicly available measurements from NREL in Denver, ${ }^{65}$ measured every $5 \mathrm{~min}$ throughout the year 2018. Measurements of the direct solar spectra are readily available (SRRL PGS-100 Direct Normal). The diffuse solar spectra could be calculated using the direct spectra, the global horizontal measurements (SRRL WISER Global or Direct) and the azimuth angle of the sun. The temperature of the cell, used in the calculation of $J_{0}$, was approximated by the locally measured temperature (Dry Bulb Temperature (deck)). For each spectrum and cell temperature, a detailed balance model is used to determine $J_{s c}$ and $J_{0}$, and from these the maximum efficiency is calculated. Simulation details can be found in the supporting information. The results are presented in Figure 7,

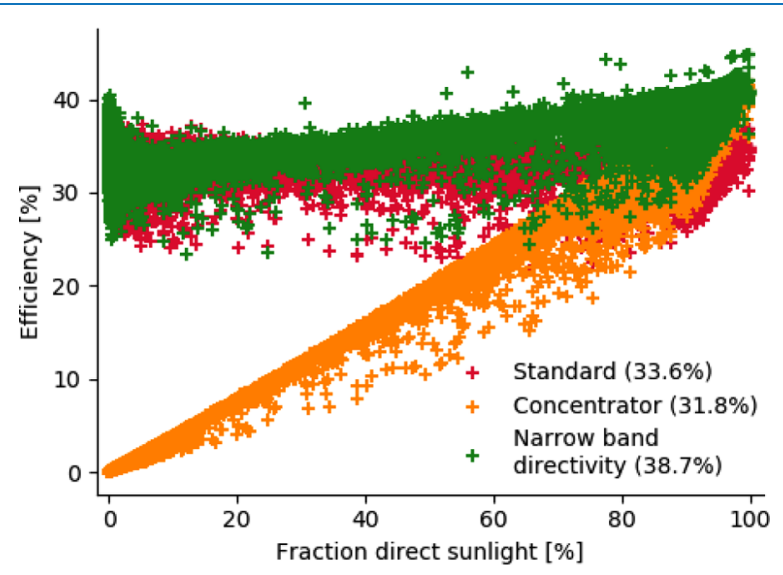

Figure 7. Performance of a conventional concentrator system and narrow band directivity system compared to a cell without concentration. The efficiency is plotted as a function of the fraction of direct sunlight and the simulations are based on measured solar spectra. ${ }^{65}$ The values in brackets indicate the average efficiency over the year. The concentrator reduces the efficiency by $2.7 \%$, while the narrow band directive structures increase efficiency by absolute $7.7 \%$.

showing the increase in efficiency compared to the cell without concentration or directivity, plotted versus fraction of direct sunlight. For low fractions of direct sunlight, macroscopic concentration performs very poorly, as expected from eq 10 . The efficiency based on annual energy yield is $31.8 \%$, which is a relative decrease of $5 \%$ compared to the standard cell (33.6\%). This difference is smaller than it might seem from Figure 7. This is because, in general, low fractions of direct sunlight correspond to low total incoming power, so the absolute loss in generated power is limited. The narrow band 

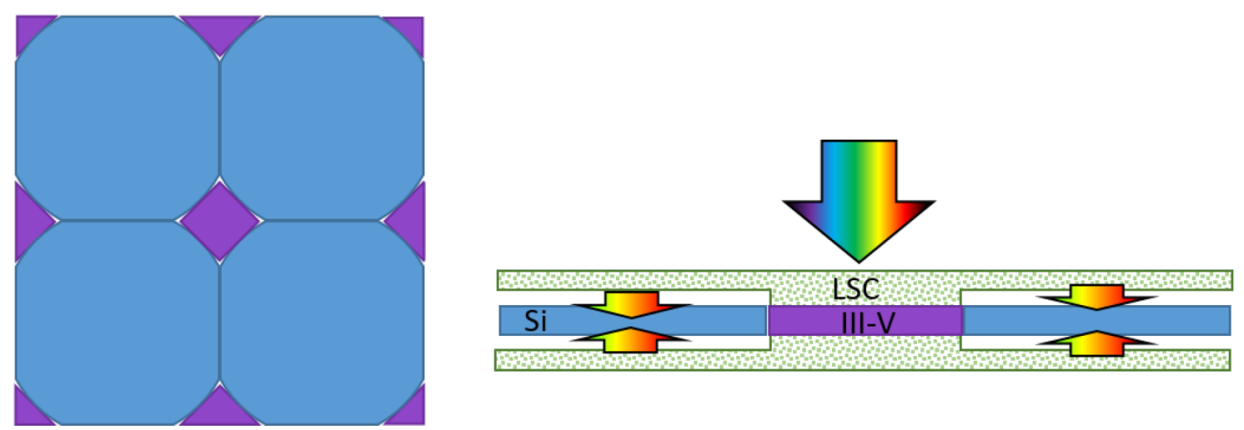

Figure 8. Schematic layout of the LSC-Si tandem configuration, where the high band gap cells sit in the gaps between the silicon wafers. The connection between the LSC and the small solar cells must be index matched, such that the waveguided light leaks out and couples into the cell.

directivity concept outperforms the standard cell over the whole range, and the annual energy conversion efficiency is $38.7 \%$, a relative increase of $15 \%$ compared to the standard cell. This concept does not suffer from high fractions of diffuse sunlight, which shows the importance of having high directivity only over a narrow bandwidth.

There are still several challenges that need to be solved to realize this concept. As suggested in the section on matching the angles, directive nanostructures can be made in different ways, both from the semiconductor itself or from a dielectric nanolenses on top of the semiconductor. A cheap and fast fabrication technique for directive structures has to be found. A question is whether it is possible to obtain the desired directivity with a texture on a planar film, or by using diffractive effects. Another question is what happens when directive structures are placed in an array and the enhanced absorption cross sections start to overlap at certain wavelengths or angles. Also fundamental questions need to be solved, to show if there is a limit on directivity in nanostructures or if there is a trade off between directivity, LDOS, and light outcoupling. It has to be shown, both theoretically and experimentally, whether the required emission patterns can be achieved. High directivity into a narrow cone requires accurate two-axis solar tracking: one "fast" moving rotation to follow the arc of the sun throughout the day and one slow rotation to adjust the axis throughout the year. If a directive structure can be made that emits into a band matching the arc of the sun, tracking throughout the day could be eliminated, allowing for the widely available and inexpensive single axis tracking to be used. These questions and more require further research to nanoscale directivity.

Bifacial LSC-Si Tandem Device. The second concept aims at combining cheap and well established silicon solar cells with new techniques. We propose a bifacial tandem configuration where a high band gap LSC is placed on both sides of a bifacial silicon panel. This concept combines the existing design of silicon cells with a yet to be developed LSC and solves several problems at the same time.

Currently, the industry of silicon cells is moving toward bifacial cells, which can also absorb sunlight from the back side. This can give a significant increase in energy output per panel, especially in regions with large fractions of diffuse sunlight. To increase energy output even further, the efficiency has to be increased. As discussed in relation to Figure 2, silicon solar cells perform close to their limit, and improving efficiency requires new measures. The biggest gain can be found in going to a tandem configuration, with a high band gap material on top of the silicon. As mentioned before, conventional tandem cells are expensive to fabricate and suffer from current matching. Using an LSC can potentially be a very cheap solution. Standard silicon solar cells already have an encapsulation layer that could serve a dual purpose as an LSC, without adding a fabrication step to the module assembly. Currently existing LSCs suffer from low efficiency, and this is the main challenge that might be solved with directional emission from nanostructures in the LSC.

A typical solar cell module consists of an array of silicon wafers. For monocrystalline silicon, these wafers are cut from a cylindrical ingot. The packing efficiency in a panel is increased by cutting the circular wafers into squares. To limit the waste of material, the squares are cut slightly too big, which leads to "missing" corners that are typically visible on the modules. We propose to place the high band gap cells of the LSC in these empty squares of the module. For standard silicon cells used in industry (commonly called 6 in. wafers), the missing corners form a fraction of $0.6 \%$ of the total area. This gives a concentration factor of 170 for the LSC. In Figure 8, the layout is shown schematically. For the small, high band gap cells, high efficiency III-V materials can be used. These are expensive, but the concentration of the LSC reduces the price by a factor of 170 . By placing them in between the c-Si cells, there is no need for lattice matching or wafer bonding.

Aligned directive nanostructures, with an absorption cross section as depicted in Figure 6, are used to optimize emission into the waveguide and to minimize reabsorption. The exact requirements on the absorption cross section depend on several parameters, like the refractive index of the waveguide (and thus the escape cone), the PLQY of the emitters, the Stokes shift, and the scattering cross section. Fully modeling this is beyond the scope of this paper. A simple parameter that captures several effects is the internal efficiency $Q_{\text {int }}$ that gives the ratio of the number of photons that reach the LSC solar cells compared to the number of photons absorbed in the LSC (sometimes also called the optical quantum efficiency in the LSC community). This parameter accounts for the major loss mechanisms in an LSC: photons escaping the waveguide and photons lost due to nonradiative recombination, including the effects of reabsorption along the way. We use this parameter to take into account the directive emission in the modeling of the performance of the LSC-tandem concept: a high $Q_{\text {int }}$ corresponds to high directivity, because this reduces reabsorption and escaping of photons.

To simulate the performance of this concept, several parameters have to be quantified. For the bifacial silicon cell, a PLQY of $1 \%$ and a stepwise band gap of $1.1 \mathrm{eV}$ are assumed. The optimum band gap of the LSC was found to be $1.75 \mathrm{eV}$ 
through optimization with measured solar spectra. The high band gap cells are modeled to have a PLQY of $85 \%$. Different values of $Q_{\text {int }}$ have been evaluated. If less than three-quarters of the photons absorbed in the LSC are collected, it performs worse than the current matched tandem. If less than half of the photons are collected, the performance is worse than only the bifacial silicon cell. Here we model the performance of a system with $Q_{\text {int }}=90 \%$. We assume that the back side of the bifacial cell receives $75 \%$ of the diffuse sunlight. For a bifacial silicon panel without LSC this gives a yearly increase in energy output of $15 \%$, compared to a monofacial cell. Some long running field tests with bifacial cells have shown an increase between $10 \%$ and $20 \%$, so the increase of $15 \%$ is a realistic approximation. ${ }^{66}$ The performance of the LSC-Si tandem is compared with a current matched tandem, with the same bifacial silicon bottom cell, but no tandem on the back. The top cell has again a band gap of $1.75 \mathrm{eV}$ and a PLQY of $85 \%$.

With these assumptions, the performance is again calculated based on the measured solar spectra ${ }^{65}$ by simulating the tandem devices in a detailed balance model (simulation details in SI). The results of the simulation are shown in Figure 9,

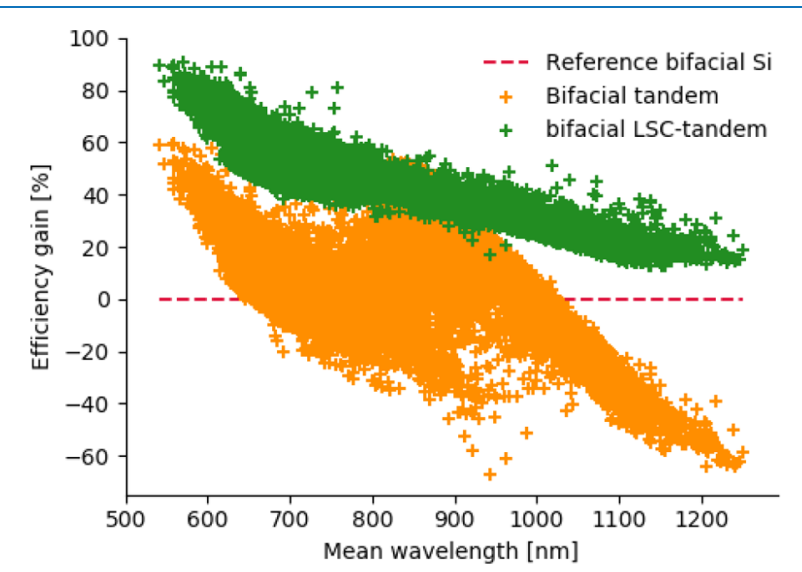

Figure 9. Relative performance of a bifacial current matched tandem and a bifacial LSC-tandem configuration compared to a bifacial silicon solar cell. The relative performance is plotted as a function of the mean wavelength in the spectrum and the simulations are based on measured solar spectra. ${ }^{65}$ The current matched tandem and the LSC tandem show an absolute increase of $9.2 \%$ and $13.3 \%$ in power generation compared to the bifacial silicon cell, respectively.

where the performance is plotted relative to a single junction bifacial cell. In this case, the efficiency is plotted versus the mean wavelength of the spectrum. Spectral shifts reduce the performance of current matched tandem, because the current is determined by the minimum current in either of the two cells. If the spectrum is red-shifted, the current in the high band gap cell decreases. The blue-shifted spectra correspond to high fractions of diffuse sunlight. In this case, the reduced current in the $\mathrm{Si}$ bottom cell due to the blue-shift is compensated with the contribution of diffuse sunlight on the back side, and the efficiency remains high. The yearly energy output compared to standard bifacial panel is increased by an absolute $9.2 \%$, which is a $28 \%$ relative increase.

The bifacial LSC-tandem configuration exceeds the performance of the two others at almost every spectrum. The yearly energy output is increased by $13.3 \%$ compared to the single junction bifacial cell and $4.1 \%$ compared to the current matched tandem, relative increases of $41 \%$ and $10 \%$, respectively. It benefits from the increased efficiency of a tandem device, without current losses and with less complex manufacturing. This shows the great potential of optimized LSC-tandem configurations.

The performance of the systems is given as a relative efficiency compared to a reference bifacial cell. We want to mention that the efficiency of a bifacial device is ill-defined without specifying the environment around the cell, PV packing density, and other geometric factors. The great benefit of bifacial configurations is the fact that they can harvest diffuse sunlight that is scattered from elsewhere. When the efficiency is calculated based on the incident sunlight per unit area, this can lead to apparently very high efficiencies at high fractions of diffuse sunlight, because light is effectively harvested from a larger area. This leads to seemingly unrealistic results when reporting absolute efficiencies, and therefore, it is more insightful to look at the relative performance of the three systems.

For this concept, the same questions apply regarding directive nanostructures as for the first design. A detailed modeling of the aligned nanolenses in a waveguide must be done to show whether asymmetric transmission can be achieved. One possible problem could be the scattering of light in undesired directions, but some preliminary modeling showed that the transmission of directive nanolenses is high and that less than $10 \%$ of the light incident on the back (low absorption) side is scattered backward. Furthermore, techniques need to be developed to make waveguides with aligned nanostructures and couple them to solar cells.

\section{CONCLUSION}

In this Perspective, we gave an overview of how nanomaterials can increase solar cell efficiency. Nanomaterials have a great potential due to their strong and tunable interaction with light, originating from their size being comparable to the wavelength of light. This allows for controlling the emission event itself, instead of manipulating the light after it has been emitted, which is a key difference compared to macroscopic approaches. In this Perspective, we covered the different aspects in which nanomaterials can play a role in increasing solar cell efficiency. To do so, we took a step back and looked at the fundamental properties needed to make a good solar cell. From thermodynamics, we learned that an ideal solar cell in open circuit should emit all light back to the sun. This shows the importance of good light management, not only to optimizing absorption, but also making good, defect-free materials and applying nanophotonic engineering to accurately control the emission. By comparing a typical silicon solar cell to the idealized thermodynamic model, we identified three differences in the emitted light: the spectral bandwidth, the intensity, and the angular emission profile. Each of these cause a reduction in $V_{\mathrm{oc}}$, while $J_{\mathrm{sc}}$ is only mildly affected. This is also seen in record solar cells: while $J_{\text {sc }}$ approaches the theoretical limit, $V_{\text {oc }}$ is typically lagging behind. This Perspective focuses on ways to increase $V_{\text {oc }}$ with nanomaterials, because this field has only recently emerged and is rapidly developing.

For each of the three loss mechanisms, we gave an overview of how nanomaterials can help in reducing the losses. We have pointed out what has been achieved so far and what the remaining challenges are. Finally, we applied this knowledge in two conceptual solar cell designs. We showed that, with nanostructures that reduce the cone of emission while maintaining sensitive to diffuse light, the performance in 
terrestrial application can be increased by $15 \%$. A conventional, macroscopic solution will actually decrease performance by $8 \%$ due to its low performance in the presence of diffuse sunlight. A bifacial LSC-tandem configuration based on nanophotonic structures can increase performance compared to a bifacial silicon cell by $41 \%$, while a more conventional current matched tandem gives an increase of $28 \%$. Further development in designing and fabricating nanostructures for accurately optimized light management has the potential for contributing to high efficiency solar cells at low cost.

\section{ASSOCIATED CONTENT}

\section{SI Supporting Information}

The Supporting Information is available free of charge at https://pubs.acs.org/doi/10.1021/acsphotonics.0c00152.

Derivation, including the assumptions made in eqs 9 and 10, and details of solar cell efficiency model (PDF)

\section{AUTHOR INFORMATION}

\section{Corresponding Author}

Erik C. Garnett - AMOLF, Center for Nanophotonics, NoordHolland NL 1098XG, Amsterdam; 이이.org/0000-00029158-8326; Phone: 0031207547100; Email: e.garnett@ amolf.nl

\section{Author \\ Julia S. van der Burgt - AMOLF, Center for Nanophotonics, Noord-Holland NL 1098XG, Amsterdam}

Complete contact information is available at: https://pubs.acs.org/10.1021/acsphotonics.0c00152

\section{Notes}

The authors declare no competing financial interest.

\section{ACKNOWLEDGMENTS}

The authors acknowledge the helpful discussions with Thomas Kirchartz and thank him for his feedback. We thank Bruno Ehrler for a careful reading of the manuscipt. This work is part of the research programme Mat4Sus, which is financed by The Netherlands Organisation for Scientific Research (NWO). The work has been carried out at Amolf, an NWO-funded institute.

\section{REFERENCES}

(1) IRENA (2018), Renewable Power Generation Costs in 2017. International Renewable Energy Agency, Abu, Dhabi, 2018, ISBN 978-92-9260-040-2.

(2) Chung, D.; Davidson, C.; Fu, R.; Ardani, K.; Margolis, R. U.S. Photovoltaic Prices and Cost Breakdowns: Q1 2015 Benchmarks for Residential, Commercial, and Utility-Scale Systems. National Renewable Energy Laboratory 2015.

(3) International Energy Agency IEA, Technology Roadmap - Solar Photovoltaic Energy. International Energy Agency, Paris, 2014, https://www.iea.org/reports/technology-roadmap-solar-photovoltaicenergy-2014.

(4) Polman, A.; Knight, M.; Garnett, E. C.; Ehrler, B.; Sinke, W. C. Photovoltaic materials: Present efficiencies and future challenges. Science 2016, 352 (6283), aad4424.

(5) Brongersma, M. L.; Cui, Y.; Fan, S. Light management for photovoltaics using high-index nanostructures. Nat. Mater. 2014, 13 (5), 451-460.

(6) Atwater, H.; Polman, A.; Kosten, E.; Callahan, D.; Spinelli, P.; Eisler, C.; Escarra, M.; Warmann, E.; Flowers, C. Nanophotonic design principles for ultrahigh efficiency photovoltaics. AIP Conf. Proc. 2012, 1519, 17-21.
(7) Koenderink, A. F. Single-Photon Nanoantennas. ACS Photonics 2017, 4 (4), 710-722.

(8) Mann, S. A.; Grote, R. R.; Osgood, R. M.; Alù, A.; Garnett, E. C. Opportunities and Limitations for Nanophotonic Structures to Exceed the Shockley-Queisser Limit. ACS Nano 2016, 10 (9), $8620-8631$.

(9) Shockley, W.; Queisser, H. J. Detailed Balance Limit of Efficiency of pn Junction Solar Cells. J. Appl. Phys. 1961, 32 (3), 510519.

(10) Araújo, G. L.; Martí, A. Absolute limiting efficiencies for photovoltaic energy conversion. Sol. Energy Mater. Sol. Cells 1994, 33, 213-240.

(11) Kosten, E. D.; Newman, B. K.; Lloyd, J. V.; Polman, A.; Atwater, H. A. Limiting light escape angle in silicon photovoltaics: Ideal and realistic cells. IEEE Journal of Photovoltaics 2015, 5 (1), 6169

(12) Peters, M.; Goldschmidt, J. C.; Kirchartz, T.; Bläsi, B. The photonic light trap-Improved light trapping in solar cells by angularly selective filters. Sol. Energy Mater. Sol. Cells 2009, 93, 1721-1727.

(13) Peters, M.; Goldschmidt, J. C.; Löper, P.; Groß, B.; Üpping, J.; Dimroth, F.; Wehrspohn, R. B.; Blasi, B. Spectrally-Selective photonic structures for PV applications. Energies 2010, 3 (2), 171-193.

(14) Martí, A.; Balenzategui, J. L.; Reyna, R. F. Photon recycling and Shockley's diode equation. J. Appl. Phys. 1997, 82 (8), 4067-4075.

(15) Cui, Y.; Van Dam, D.; Mann, S. A.; Van Hoof, N. J.; Van Veldhoven, P. J.; Garnett, E. C.; Bakkers, E. P.; Haverkort, J. E. Boosting Solar Cell Photovoltage via Nanophotonic Engineering. Nano Lett. 2016, 16 (10), 6467-6471.

(16) Zhang, D.; Gu, L.; Zhang, Q.; Lin, Y.; Lien, D. H.; Kam, M.; Poddar, S.; Garnett, E. C.; Javey, A.; Fan, Z. Increasing Photoluminescence Quantum Yield by Nanophotonic Design of QuantumConfined Halide Perovskite Nanowire Arrays. Nano Lett. 2019, 19 (5), 2850-2857.

(17) Koenderink, A. F.; Alu, A.; Polman, A. Nanophotonics: Shrinking light-based technology. Science 2015, 348 (6234), 516-521.

(18) De Vos, A.; Pauwels, H. On the thermodynamic limit of photovoltaic energy conversion. Appl. Phys. 1981, 25 (2), 119-125.

(19) Kirchhoff, G. Ueber das Verhältniss zwischen dem Emissionsvermögen und dem Absorptionsvermögen der Körper für Wärme und Licht. Ann. Phys. 1860, 185 (2), 275-301.

(20) Kirchartz, T.; Rau, U. What Makes a Good Solar Cell? Adv. Energy Mater. 2018, 8 (28), 1703385.

(21) Green, M. A.; Bremner, S. P. Energy conversion approaches and materials for high-efficiency photovoltaics. Nat. Mater. 2017, 16 (1), 23-34.

(22) Guillemoles, J. F.; Kirchartz, T.; Cahen, D.; Rau, U. Guide for the perplexed to the Shockley-Queisser model for solar cells. Nat. Photonics 2019, 13 (8), 501-505.

(23) Green, M. A.; Dunlop, E. D.; Levi, D. H.; Hohl-Ebinger, J.; Yoshita, M.; Ho-Baillie, A. W. Solar cell efficiency tables (version 54). Prog. Photovoltaics 2019, 27 (7), 565-575.

(24) Garnett, E. C.; Brongersma, M. L.; Cui, Y.; McGehee, M. D. Nanowire Solar Cells. Annu. Rev. Mater. Res. 2011, 41 (1), 269-295.

(25) Savin, H.; Repo, P.; Von Gastrow, G.; Ortega, P.; Calle, E.; Garín, M.; Alcubilla, R. Black silicon solar cells with interdigitated back-contacts achieve $22.1 \%$ efficiency. Nat. Nanotechnol. 2015, 10, 624-628.

(26) Oh, J.; Yuan, H. C.; Branz, H. M. An 18.2\%-efficient blacksilicon solar cell achieved through control of carrier recombination in nanostructures. Nat. Nanotechnol. 2012, 7, 743-748.

(27) Black, L. E.; Cavalli, A.; Verheijen, M. A.; Haverkort, J. E.; Bakkers, E. P.; Kessels, W. M. M. Effective Surface Passivation of InP Nanowires by Atomic-Layer-Deposited $\mathrm{Al}_{2} \mathrm{O}_{3}$ with $\mathrm{PO}_{x}$ Interlayer. Nano Lett. 2017, 17 (10), 6287-6294.

(28) Zhang, Q.; Tavakoli, M. M.; Gu, L.; Zhang, D.; Tang, L.; Gao, Y.; Guo, J.; Lin, Y.; Leung, S. F.; Poddar, S.; Fu, Y.; Fan, Z. Efficient metal halide perovskite light-emitting diodes with significantly improved light extraction on nanophotonic substrates. Nat. Commun. 2019, 10, 727. 
(29) Isabella, O.; Jäger, K.; Smets, S.; van Swaaij, R.; Zeman, M. Generation and recombination of electron-hole pairs. Solar Energy: The Physics and Engineering of Photovoltaic Conversion, Technologies and Systems; UIT Cambridge, 2016; pp 65-82.

(30) Rau, U.; Paetzold, U. W.; Kirchartz, T. Thermodynamics of light management in photovoltaic devices. Phys. Rev. B: Condens. Matter Mater. Phys. 2014, 90 (3), 035211.

(31) Richter, J. M.; Abdi-Jalebi, M.; Sadhanala, A.; Tabachnyk, M.; Rivett, J. P.; Pazos-Outón, L. M.; Gödel, K. C.; Price, M.; Deschler, F.; Friend, R. H. Enhancing photoluminescence yields in lead halide perovskites by photon recycling and light out-coupling. Nat. Commun. 2016, 7, 13941.

(32) Anttu, N.; Kivisaari, P.; Chen, Y. Tailored emission to boost open-circuit voltage in solar cells. Journal of Physics Communications 2019, 3 (5), No. 055009.

(33) Mann, S. A.; Oener, S. Z.; Cavalli, A.; Haverkort, J. E.; Bakkers, E. P.; Garnett, E. C. Quantifying losses and thermodynamic limits in nanophotonic solar cells. Nat. Nanotechnol. 2016, 11, 1071-1075.

(34) Sandhu, S.; Yu, Z.; Fan, S. Detailed Balance Analysis and Enhancement of Open-Circuit Voltage in Single-Nanowire Solar Cells. Nano Lett. 2014, 14 (2), 1011-1015.

(35) Rau, U. Reciprocity relation between photovoltaic quantum efficiency and electroluminescent emission of solar cells. Phys. Rev. B: Condens. Matter Mater. Phys. 2007, 76 (8), No. 085303.

(36) Braly, I. L.; Stoddard, R. J.; Rajagopal, A.; Jen, A. K.; Hillhouse, $\mathrm{H}$. W. Photoluminescence and Photoconductivity to Assess Maximum Open-Circuit Voltage and Carrier Transport in Hybrid Perovskites and Other Photovoltaic Materials. J. Phys. Chem. Lett. 2018, 9 (13), 3779-3792.

(37) Delamarre, A.; Lombez, L.; Guillemoles, J. F. Contactless mapping of saturation currents of solar cells by photoluminescence. Appl. Phys. Lett. 2012, 100 (13), 131108.

(38) ESMAP, Global Solar Atlas. https://globalsolaratlas.info/map, accessed October 2019. Data obtained from the Global Solar Atlas 2.0, a free, web-based application is developed and operated by the company Solargis s.r.o. on behalf of the World Bank Group, utilizing Solargis data, with funding provided by the Energy Sector Management Assist.

(39) Kraus, T.; Höhn, O.; Hauser, H.; Bläsi, B. Optoelectronic simulation of GaAs solar cells with angularly selective filters. J. Appl. Phys. 2014, 115 (5), 053103.

(40) Kosten, E. D.; Kayes, B. M.; Atwater, H. A. Experimental demonstration of enhanced photon recycling in angle-restricted GaAs solar cells. Energy Environ. Sci. 2014, 7 (6), 1907-1912.

(41) Peters, M.; Goldschmidt, J. C.; Bläsi, B. Angular confinement and concentration in photovoltaic converters. Sol. Energy Mater. Sol. Cells 2010, 94, 1393-1398.

(42) Ulbrich, C.; Fahr, S.; Üpping, J.; Peters, M.; Kirchartz, T.; Rockstuhl, C.; Wehrspohn, R.; Gombert, A.; Lederer, F.; Rau, U. Directional selectivity and ultra-light-trapping in solar cells. Phys. Status Solidi A 2008, 205 (12), 2831-2843.

(43) Curto, A. G.; Volpe, G.; Taminiau, T. H.; Kreuzer, M. P.; Quidant, R.; van Hulst, N. F. Unidirectional Emission of a Quantum Dot Coupled to a Nanoantenna. Science 2010, 329 (5994), 930-933.

(44) Aouani, H.; Mahboub, O.; Devaux, E.; Rigneault, H.; Ebbesen, T. W.; Wenger, J. Plasmonic antennas for directional sorting of fluorescence emission. Nano Lett. 2011, 11 (6), 2400-2406.

(45) Lozano, G.; Louwers, D. J.; Rodríguez, S. R. K.; Murai, S.; Jansen, O. T. A.; Verschuuren, M. A.; Gómez Rivas, J. Plasmonics for solid-state lighting: Enhanced excitation and directional emission of highly efficient light sources. Light: Sci. Appl. 2013, 2, No. e66.

(46) Yu, N.; Capasso, F. Flat optics with designer metasurfaces. Nat. Mater. 2014, 13 (2), 139-150.

(47) Krasnok, A. E.; Miroshnichenko, A. E.; Belov, P. a.; Kivshar, Y. S. All-dielectric optical nanoantennas. Opt. Express 2012, 20 (18), 20599-20604.

(48) Andersen, S. K.; Bogdanov, S.; Makarova, O.; Xuan, Y.; Shalaginov, M. Y.; Boltasseva, A.; Bozhevolnyi, S. I.; Shalaev, V. M.
Hybrid Plasmonic Bullseye Antennas for Efficient Photon Collection. ACS Photonics 2018, 5 (3), 692-698.

(49) Filonov, D. S.; Krasnok, A. E.; Slobozhanyuk, A. P.; Kapitanova, P. V.; Nenasheva, E. A.; Kivshar, Y. S.; Belov, P. A. Experimental verification of the concept of all-dielectric nanoantennas. Appl. Phys. Lett. 2012, 100 (20), 201113.

(50) Khorasaninejad, M.; Capasso, F. Metalenses: Versatile multifunctional photonic components. Science 2017, 358 (6367), No. eaam 8100 .

(51) Atwater, H. A.; Polman, A. Plasmonics for improved photovoltaic devices. Nat. Mater. 2010, 9 (3), 205-213.

(52) Johlin, E.; Mann, S. A.; Kasture, S.; Koenderink, A. F.; Garnett, E. C. Broadband highly directive 3D nanophotonic lenses. Nat. Commun. 2018, 9, 4742.

(53) Holtus, T.; Helmbrecht, L.; Hendrikse, H. C.; Baglai, I.; Meuret, S.; Adhyaksa, G. W.; Garnett, E. C.; Noorduin, W. L. Shapepreserving transformation of carbonate minerals into lead halide perovskite semiconductors based on ion exchange/insertion reactions. Nat. Chem. 2018, 10 (7), 740-745.

(54) Scott, R.; Heckmann, J.; Prudnikau, A. V.; Antanovich, A.; Mikhailov, A.; Owschimikow, N.; Artemyev, M.; Climente, J. I.; Woggon, U.; Grosse, N. B.; Achtstein, A. W. Directed emission of $\mathrm{CdSe}$ nanoplatelets originating from strongly anisotropic 2D electronic structure. Nat. Nanotechnol. 2017, 12, 1155-1161.

(55) Fieramosca, A.; De Marco, L.; Passoni, M.; Polimeno, L.; Rizzo, A.; Rosa, B. L.; Cruciani, G.; Dominici, L.; De Giorgi, M.; Gigli, G.; Andreani, L. C.; Gerace, D.; Ballarini, D.; Sanvitto, D. Tunable Outof-Plane Excitons in 2D Single-Crystal Perovskites. ACS Photonics 2018, 5 (10), 4179-4185.

(56) Needell, D. R.; Ilic, O.; Bukowsky, C. R.; Nett, Z.; Xu, L.; He, J.; Bauser, H.; Lee, B. G.; Geisz, J. F.; Nuzzo, R. G.; Alivisatos, A. P.; Atwater, H. A. Design criteria for micro-optical tandem luminescent solar concentrators. IEEE Journal of Photovoltaics 2018, 8 (6), 15601567.

(57) Wu, K.; Li, H.; Klimov, V. I. Tandem luminescent solar concentrators based on engineered quantum dots. Nat. Photonics 2018, 12, 105-110.

(58) Goldschmidt, J. C.; Peters, M.; Gutmann, J.; Steidl, L.; Zentel, R.; Bläsi, B.; Hermle, M. Increasing fluorescent concentrator light collection efficiency by restricting the angular emission characteristic of the incorporated luminescent material: the 'Nano-Fluko' concept. Proc. SPIE 2010, 7725, 77250S.

(59) Ehrler, B.; Musselman, K. P.; Böhm, M. L.; Friend, R. H.; Greenham, N. C. Hybrid pentacene/a-silicon solar cells utilizing multiple carrier generation via singlet exciton fission. Appl. Phys. Lett. 2012, 101 (15), 153507.

(60) Kirchartz, T.; Rau, U. Modeling charge carrier collection in multiple exciton generating PbSe quantum dots. Thin Solid Films 2009, 517 (7), 2438-2442.

(61) Li, M.; Begum, R.; Fu, J.; Xu, Q.; Koh, T. M.; Veldhuis, S. A.; Grätzel, M.; Mathews, N.; Mhaisalkar, S.; Sum, T. C. Low threshold and efficient multiple exciton generation in halide perovskite nanocrystals. Nat. Commun. 2018, 9, 4197.

(62) de Weerd, C.; Gomez, L.; Capretti, A.; Lebrun, D. M.; Matsubara, E.; Lin, J.; Ashida, M.; Spoor, F. C.; Siebbeles, L. D.; Houtepen, A. J.; Suenaga, K.; Fujiwara, Y.; Gregorkiewicz, T. Efficient carrier multiplication in $\mathrm{CsPbI}_{3}$ perovskite nanocrystals. Nat. Commun. 2018, 9, 4199.

(63) Dorodnyy, A.; Alarcon-Lladó, E.; Shklover, V.; Hafner, C.; Fontcuberta I Morral, A.; Leuthold, J. Efficient Multiterminal Spectrum Splitting via a Nanowire Array Solar Cell. ACS Photonics 2015, 2 (9), 1284-1288.

(64) Mann, S. A.; Garnett, E. C. Resonant nanophotonic spectrum splitting for ultrathin multijunction solar cells. ACS Photonics 2015, 2 (7), 816-821.

(65) Andreas, A.; Stoffel, T. NREL Solar Radiation Research Laboratory (SRRL): Baseline Measurement System (BMS); Golden, Colorado (Data). NREL Report No. DA-5500-56488. DOI: 10.5439/ 1052221. 
(66) Liang, T. S.; Pravettoni, M.; Deline, C.; Stein, J. S.; Kopecek, R.; Singh, J. P.; Luo, W.; Wang, Y.; Aberle, A. G.; Khoo, Y. S. A review of crystalline silicon bifacial photovoltaic performance characterisation and simulation. Energy Environ. Sci. 2019, 12 (1), 116-148. 